\title{
Characterizing the generalized lambda distribution by L-moments
}

\author{
Juha Karvanen \\ National Public Health Institute, \\ Mannerheimintie 166, 00300 Helsinki, Finland, \\ juha.karvanen@ktl.fi \\ Arto Nuutinen \\ University of Helsinki, \\ Väinö Auerin katu 1, 00560 Helsinki, Finland
}

September 9, 2018

\begin{abstract}
The generalized lambda distribution (GLD) is a flexible four parametric distribution with many practical applications. L-moments of the GLD can be expressed in closed form and are good alternatives for the central moments. In this paper, we present the L-moments of the GLD up to an arbitrary order and study which values of Lskewness and L-kurtosis can be achieved by the GLD. The boundaries of L-skewness and L-kurtosis are derived analytically in the symmetric case and calculated numerically in the general case. In addition, the contours of L-skewness and L-kurtosis are presented as functions of the GLD parameters. It is found that with an exception of the smallest values of L-kurtosis, the GLD covers all possible pairs of L-skewness and L-kurtosis and often there are two or more distributions that share the same L-skewness and the same L-kurtosis. We present an example that demonstrates a situation where there are four GLD members with the same L-skewness and the same L-kurtosis. The results increase our knowledge on the distributions that belong to the GLD family and can be utilized in model selection and estimation.
\end{abstract}

Keywords: skewness, kurtosis, L-moment ratio diagram, method of moments, method of L-moments 


\section{Introduction}

The generalized lambda distribution (GLD) is a four parametric distribution that has been applied to various problems where a flexible parametric model for univariate data is needed. The GLD offers a large variety of skewness and kurtosis and can approximate many commonly used distributions such as normal and uniform. The applications of the GLD include e.g. option pricing (Corrado, 2001), independent component analysis (Karvanen et al., 2002), statistical process control (Pal, 2005), analysis of fatigue of materials (Bigerelle et al., 2005), measurement technology (Lampasi et al., 2005) and generation of random variables (Ramberg and Schmeiser, 1974; Karvanen, 2003; Headrick and Mugdadib, 2006).

The price for the high flexibility is high complexity. Probability density function (pdf) or cumulative distribution function (cdf) of the GLD do not exist in closed form but the distribution is defined by the inverse distribution function (Ramberg and Schmeiser, 1974)

$$
F^{-1}(u)=\lambda_{1}+\frac{u^{\lambda_{3}}-(1-u)^{\lambda_{4}}}{\lambda_{2}}
$$

where $0 \leq u \leq 1$ and $\lambda_{1}, \lambda_{2}, \lambda_{3}$ and $\lambda_{4}$ are the parameters of the GLD. Equation (11) defines a distribution if and only if (Karian et al., 1996)

$$
\frac{\lambda_{2}}{\lambda_{3} u^{\lambda_{3}-1}+\lambda_{4}(1-u)^{\lambda_{4}-1}} \geq 0 \text { for all } u \in[0,1] .
$$

Karian et al. (1996) divide the GLD into six regions on the basis of the parameters $\lambda_{3}$ and $\lambda_{4}$ that control skewness and kurtosis of the distribution. These regions are presented in Figure 1. The regions have different characteristics: the distributions in the region 3 are bounded whereas the distributions in the region 4 are unbounded; the distributions in the regions 1 and 5 are bounded on the right and the distributions in the regions 2 and 6 are bounded on the left. See e.g. (Dudewicz and Karian, 2000) or (Fournier et al., 2006) for the boundaries of the domain in each region.

The members of the GLD family have been traditionally characterized by the central moments, especially by the central moment based skewness and kurtosis (Ramberg and Schmeiser, 1974; Karian et al., 1996). L-moments (Hosking, 1990), defined as linear combinations of order statistics, are attractive alternatives for the central moments. Differently from the central moments, the L-moments of the GLD can be expressed in closed form, which allows us to derive some analytical results and makes it easy to perform 


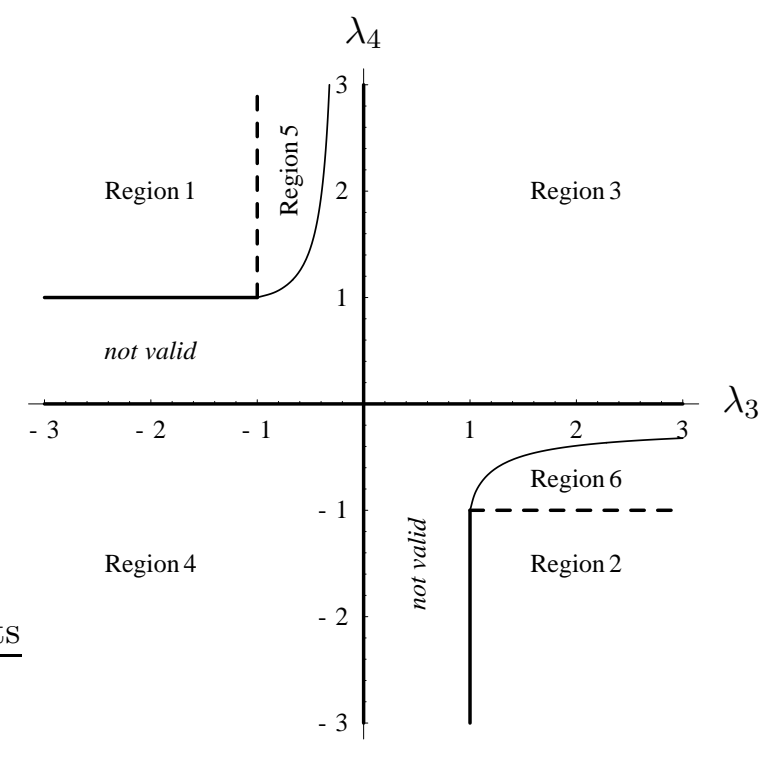

Figure 1: GLD regions as defined in (Karian et al., 1996).

numerical analysis. Analogously to method of moments, the parameters of the GLD can be estimated by method of L-moments (Karvanen et al., 2002; Asquith, 2006). Method of L-moments can be used independently or together with other estimation methods, similarly to what was done in (Fournier et al., 2006) and (Lakhany and Mausser, 2000) with method of moments, method of percentiles (Karian and Dudewicz, 1999), the least square method (Öztürk and Dale, 1985) and the starship method (King and MacGillivray, 1999).

In this paper we analyze the GLD using L-moments. In Section 2 we present the L-moments of the GLD up to an arbitrary order. In Section 3 we analyze the symmetric special case and derive the boundaries of L-kurtosis analytically and in Section 4 we consider the general case and calculate the boundaries of the GLD in the terms of L-skewness and L-kurtosis using numerical methods. The boundaries are calculated separately for each GLD region. We also calculate the contours of L-skewness and L-kurtosis as functions of $\lambda_{3}$ and $\lambda_{4}$. The examples presented illustrate the multiplicity of the GLD. Section 5 concludes the paper. 


\section{L-moments and the GLD}

The L-moment of order $r$ can be expressed as (Hosking, 1990)

$$
L_{r}=\int_{0}^{1} F^{-1}(u) P_{r-1}^{*}(u) \mathrm{d} u
$$

where

$$
P_{r-1}^{*}(u)=\sum_{k=0}^{r-1}(-1)^{r-k-1}\left(\begin{array}{c}
r-1 \\
k
\end{array}\right)\left(\begin{array}{c}
r+k-1 \\
k
\end{array}\right) u^{k}
$$

is the shifted Legendre polynomial of order $r-1$. All L-moments of a real-valued random variable exists if and only if the random variable has a finite mean and furthermore, a distribution whose mean exists, is uniquely determined by its L-moments (Hosking, 1990, 2006).

Similarly to the conventional moments, $L_{1}$ measures location and $L_{2}$ measures scaling. The higher order L-moments are usually transformed to L-moment rations

$$
\tau_{r}=\frac{L_{r}}{L_{2}} \quad r=3,4, \ldots
$$

L-skewness $\tau_{3}$ is related to the asymmetry of the distribution and L-kurtosis $\tau_{4}$ is related to the peakedness of the distribution. Differently from the conventional skewness and kurtosis, L-kurtosis and L-kurtosis are constrained by the conditions (Hosking, 1990; Jones, 2004)

$$
\begin{array}{r}
-1<\tau_{3}<1 \\
\left(5 \tau_{3}^{2}-1\right) / 4 \leq \tau_{4}<1 .
\end{array}
$$

Distributions are commonly characterized using the L-moment diagram where L-skewness is on the x-axis and L-kurtosis is on the y-axis.

The L-moments of the GLD have been presented in the literature up to order 5 (Bergevin, 1993; Karvanen et al., 2002; Asquith, 2006). We generalize the results for an arbitrary order $r$. The derivation is straightforward if we first note that

$$
\begin{aligned}
\int_{0}^{1} u^{\lambda_{3}} P_{r-1}^{*}(u) \mathrm{d} u & =\sum_{k=0}^{r-1} \frac{(-1)^{r-k-1}\left(\begin{array}{c}
r-1 \\
k
\end{array}\right)\left(\begin{array}{c}
r+k-1 \\
k
\end{array}\right)}{k+1+\lambda_{3}} \\
\int_{0}^{1}(1-u)^{\lambda_{4}} P_{r-1}^{*}(u) \mathrm{d} u & =-\int_{0}^{1}(u)^{\lambda_{4}} P_{r-1}^{*}(1-u) \mathrm{d} u \\
& =(-1)^{r} \sum_{k=0}^{r-1} \frac{(-1)^{r-k-1}\left(\begin{array}{c}
r-1 \\
k
\end{array}\right)\left(\begin{array}{c}
r+k-1 \\
k
\end{array}\right)}{k+1+\lambda_{4}}
\end{aligned}
$$


where the last equality follows from the property $P_{r-1}^{*}(1-u)=(-1)^{r-1} P_{r-1}^{*}(u)$. We obtain for $r \geq 2$

$$
\lambda_{2} L_{r}=\sum_{k=0}^{r-1}(-1)^{r-k-1}\left(\begin{array}{c}
r-1 \\
k
\end{array}\right)\left(\begin{array}{c}
r+k-1 \\
k
\end{array}\right)\left(\frac{1}{k+1+\lambda_{3}}+\frac{(-1)^{r}}{k+1+\lambda_{4}}\right) .
$$

The explicit formulas for the first six L-moments of the GLD are

$$
\begin{aligned}
L_{1}= & \lambda_{1}-\frac{1}{\lambda_{2}}\left(\frac{1}{1+\lambda_{4}}-\frac{1}{1+\lambda_{3}}\right) \\
L_{2} \lambda_{2}= & -\frac{1}{1+\lambda_{3}}+\frac{2}{2+\lambda_{3}}-\frac{1}{1+\lambda_{4}}+\frac{2}{2+\lambda_{4}} \\
L_{3} \lambda_{2}= & \frac{1}{1+\lambda_{3}}-\frac{6}{2+\lambda_{3}}+\frac{6}{3+\lambda_{3}}-\frac{1}{1+\lambda_{4}}+\frac{6}{2+\lambda_{4}}-\frac{6}{3+\lambda_{4}} \\
L_{4} \lambda_{2}= & -\frac{1}{1+\lambda_{3}}+\frac{12}{2+\lambda_{3}}-\frac{30}{3+\lambda_{3}}+\frac{20}{4+\lambda_{3}} \\
& -\frac{1}{1+\lambda_{4}}+\frac{12}{2+\lambda_{4}}-\frac{30}{3+\lambda_{4}}+\frac{20}{4+\lambda_{4}} \\
L_{5} \lambda_{2}= & \frac{1}{1+\lambda_{3}}-\frac{20}{2+\lambda_{3}}+\frac{90}{3+\lambda_{3}}-\frac{140}{4+\lambda_{3}}+\frac{70}{5+\lambda_{3}} \\
- & \frac{1}{1+\lambda_{4}}+\frac{20}{2+\lambda_{4}}-\frac{90}{3+\lambda_{4}}+\frac{140}{4+\lambda_{4}}-\frac{70}{5+\lambda_{4}} \\
L_{6} \lambda_{2}= & -\frac{1}{1+\lambda_{3}}+\frac{30}{2+\lambda_{3}}-\frac{210}{3+\lambda_{3}}+\frac{560}{4+\lambda_{3}}-\frac{630}{5+\lambda_{3}}+\frac{252}{6+\lambda_{3}} \\
& -\frac{1}{1+\lambda_{4}}+\frac{30}{2+\lambda_{4}}-\frac{210}{3+\lambda_{4}}+\frac{560}{4+\lambda_{4}}-\frac{630}{5+\lambda_{4}}+\frac{252}{6+\lambda_{4}} .
\end{aligned}
$$

Note that the results in (Asquith, 2006) and (Bergevin, 1993) are the same but expressed in a different form.

The mean of GLD and therefore also all L-moments exist if $\lambda_{3}, \lambda_{4}>-1$. This implies that the characterization by L-moments covers the regions 3,5 and 6 and to the subset of the region 4 where $\lambda_{3}, \lambda_{4}>-1$.

\section{The L-moments of the GLD in the symmetric special case}

In this section we consider the special case $\lambda_{3}=\lambda_{4}$ which defines symmetric distributions. The symmetric distributions can be bounded (in the region 3 ) or unbounded (in the region 4). Applying the condition $\lambda_{3}=\lambda_{4}$ to equations 
(13) and (14) we find that $\tau_{3}=0$ and

$$
\tau_{4}=\frac{\lambda_{3}^{2}-3 \lambda_{3}+2}{\lambda_{3}^{2}+7 \lambda_{3}+12}, \quad \lambda_{3}=\lambda_{4}>-1 .
$$

In Figure $3 \tau_{4}$ is plotted as a function of $\lambda_{3}$. The value of $\tau_{4}$ approaches to 1 when $\lambda_{3}$ approaches to $\infty$. The minimum of $\tau_{4}$ is found to be

$$
\frac{12-5 \sqrt{6}}{12+5 \sqrt{6}} \approx-0.0102
$$

and is obtained when $\lambda_{3}=-1+\sqrt{6}$.

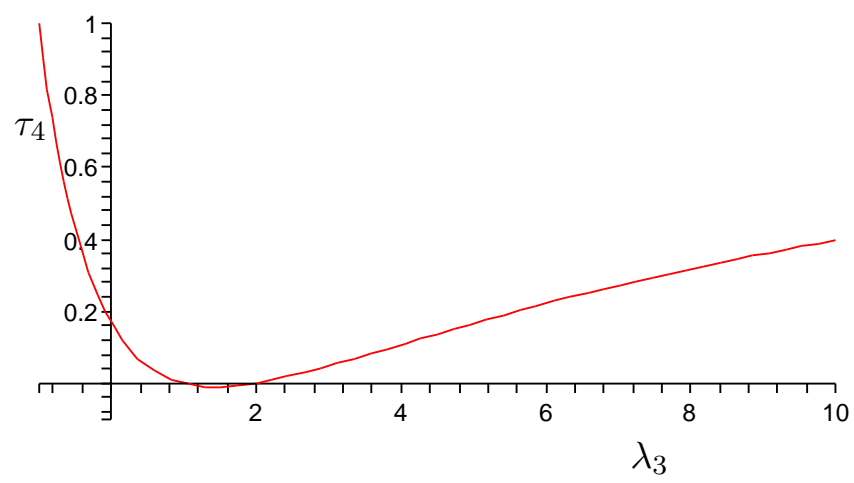

Figure 2: L-kurtosis $\tau_{4}\left(\lambda_{3}\right)$ for symmetric distributions $\lambda_{3}=\lambda_{4}$.

In the symmetric case it is also possible to solve $\lambda_{3}$ and $\lambda_{4}$ as functions of $\tau_{4}$ (Karvanen et al., 2002)

$$
\lambda_{4}=\lambda_{3}=\frac{3+7 \tau_{4} \pm \sqrt{1+98 \tau_{4}+\tau_{4}^{2}}}{2\left(1-\tau_{4}\right)} .
$$

It follows that if $\tau_{4}>1 / 6$ there are symmetric GLD members available from both the region 3 and the region 4 . If

$$
\frac{12-5 \sqrt{6}}{12+5 \sqrt{6}}<\tau_{4} \leq \frac{1}{6}
$$

there are two GLD members available from the region 3 and if

$$
\tau_{4}<\frac{12-5 \sqrt{6}}{12+5 \sqrt{6}}
$$


there are no symmetric GLD members available. Examples of symmetric GLDs sharing the same $\tau_{4}$ are presented in Figure 3. In Figure 3(a) the value of $\tau_{4}$ is set to be equal to $\tau_{4}$ of normal distribution. The both GLD members are from the region 3 and have bounded domain. The GLD of which pdf peaks higher has $\tau_{6} \approx 0.0004$ while the other GLD has $\tau_{6} \approx 0.043$ which is very near to the $\tau_{6}$ of normal distribution. The differences with normal distribution (dotted line) are visible only at the tails of the distribution. In Figure 3(b) we have $\tau_{4}=0.25$ which implies that equation (17) has one positive and one negative root. The GLD with the higher peak has bounded domain and $\tau_{6} \approx 0.016$. The other GLD with $\tau_{6} \approx 0.121$ is from the region 4 and has unbounded domain.

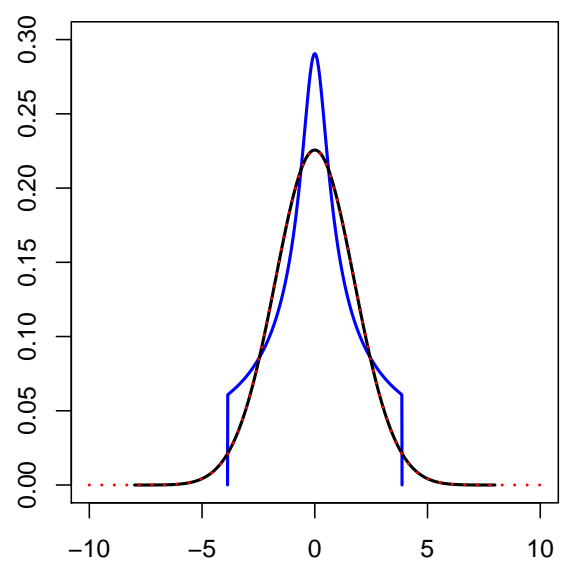

(a) $\tau_{4} \approx 0.12260\left(\tau_{4}\right.$ of normal distribution)

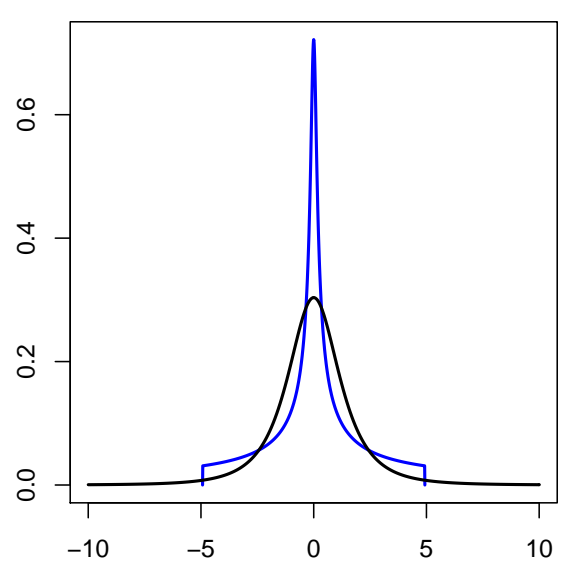

(b) $\tau_{4}=0.25$

Figure 3: Examples of symmetric GLDs sharing the same $\tau_{4}$. The pdfs of the GLDs are plotted with solid line and the pdf of normal distribution is plotted with dotted line. All distributions have $\lambda_{1}=0, \lambda_{2}=1$ and $\tau_{3}=0$.

In addition to the symmetric special case we may consider some other special cases. If we fix $\lambda_{3}=0$ we obtain

$$
\begin{aligned}
\tau_{3}\left(\lambda_{4}\right) & =\frac{1-\lambda_{4}}{\lambda_{4}+3} \\
\tau_{4}\left(\lambda_{4}\right) & =\frac{\lambda_{4}^{2}-3 \lambda_{4}+2}{\lambda_{4}^{2}+7 \lambda_{4}+12} .
\end{aligned}
$$


If we fix $\lambda_{4}=0$ we obtain

$$
\begin{aligned}
& \tau_{3}\left(\lambda_{3}\right)=\frac{\lambda_{3}-1}{\lambda_{3}+3} \\
& \tau_{4}\left(\lambda_{3}\right)=\frac{\lambda_{3}^{2}-3 \lambda_{3}+2}{\lambda_{3}^{2}+7 \lambda_{3}+12} .
\end{aligned}
$$

Interestingly, equations (23) and (25) are identical to equation (17) indicating that the $\left(\lambda_{3}, \lambda_{4}\right)$ pairs $(\lambda, \lambda),(\lambda, 0)$ and $(0, \lambda)$ lead to the same value of L-kurtosis.

\section{Boundaries of the L-moment ratios of the GLD}

In the general case it is difficult to derive analytical results and therefore we resort to numerical methods. Because the L-moments of the GLD are available in closed form, we may choose a straightforward approach and calculate the values of $\tau_{3}$ and $\tau_{4}$ for a large number of $\left(\lambda_{3}, \lambda_{4}\right)$ pairs. Naturally, the values of $\left(\lambda_{3}, \lambda_{4}\right)$ need to be chosen such a way that the essential properties of the GLD are revealed. In the region 4, we use a grid of one million points where both $\lambda_{3}$ and $\lambda_{4}$ are equally spaced in the interval $\left[-1+10^{-10}, 10^{-10}\right]$. In the region 3 we use an unequally spaced grid of 4104676 points. The grid is dense near the origin $\left(\lambda_{3}\right.$ and $\lambda_{4}$ have minimum $\left.10^{-10}\right)$ and sparse for large values $\left(\lambda_{3}\right.$ and $\lambda_{4}$ have maximum $\left.10^{12}\right)$. In the region $5, \lambda_{3}$ is equally spaced in the interval $\left[-1+10^{-10}, 0\right]$ and $\lambda_{4}$ is unequally spaced starting from -1 . The grid contains 1362429 valid points. The calculations result a large number of $\left(\tau_{3}, \tau_{4}\right)$ pairs. The results for the region 6 can be obtained from the results for the region 5 by swapping $\lambda_{3}$ and $\lambda_{4}$. The problem is to find the boundaries of the GLD in the $\left(\tau_{3}, \tau_{4}\right)$ space on the basis of these data. Let $\left(\tau_{3}[i], \tau_{4}[j]\right)$ be the image of the grid point $\left(\lambda_{3}[i], \lambda_{4}[j]\right)$, where $i$ and $j$ are the grid indexes. If the point $\left(\tau_{3}[i], \tau_{4}[j]\right)$ is not located inside the polygon defined by points $\left(\tau_{3}[i-1], \tau_{4}[j]\right),\left(\tau_{3}[i], \tau_{4}[j+1]\right),\left(\tau_{3}[i+1], \tau_{4}[j]\right)$ and $\left(\tau_{3}[i], \tau_{4}[j-1]\right)$ it is considered to be a potential boundary point. The images of the points that are located on the boundary of the grid in the $\left(\lambda_{3}, \lambda_{4}\right)$ space are also potential boundary points in the $\left(\tau_{3}, \tau_{4}\right)$ space. The actual boundaries are found combining the potential boundaries and finally it is checked that all $\left(\tau_{3}, \tau_{4}\right)$ pairs are located inside the boundaries. The calculations are made using $\mathrm{R}$ ( $\mathrm{R}$ Development Core Team, 2006) and the contributed R packages PBSmapping (Schnute et al., 2006) and gld (King, 2006) are utilized.

The boundaries are shown in Figure 4. It can be seen that region 3 covers most of the $\left(\tau_{3}, \tau_{4}\right)$ space; only the smallest values of $\tau_{4}$ are unattainable. 
Table 1: Four different GLD members with $L_{1}=0, L_{2}=1, \tau_{3}=0.4$ and $\tau_{4}=0.25$.

$\begin{array}{crrrrrr}\text { Region } & \lambda_{1} & \lambda_{2} & \lambda_{3} & \lambda_{4} & \tau_{5} & \tau_{6} \\ 3(\mathrm{a}) & 5.322 & 0.138 & 21.526 & 0.286 & 0.163 & 0.103 \\ 3(\mathrm{~b}) & -1.168 & 0.124 & 5.417 & 92.608 & -0.029 & 0.067 \\ 4 & -1.62 & -0.157 & -0.014 & -0.212 & 0.158 & 0.121 \\ 6 & -7.04 & -0.194 & 11.905 & -0.306 & 0.204 & 0.180\end{array}$

The region 4 largely overlaps with the region 3 but cannot achieve $\tau_{4}$ smaller than $1 / 6$. The region 5 and its counterpart, the region 6 , cover a rather small area of the $\left(\tau_{3}, \tau_{4}\right)$ space.

Figure 5 shows the $\left(\tau_{3}, \tau_{4}\right)$ area where there exist GLD members from the regions 3,4 and 5 , or from the regions 3,4 and 6 . To illustrate these distributions we fix $\lambda_{1}=0, \lambda_{2}=1, \tau_{3}=0.4$ and $\tau_{4}=0.25$ and seek for the solutions in the regions 3,4 and 6. Table 1 and Figure 6 present the four distributions that are found. Although the first four L-moments of the distributions are the same, the differences of the pdfs are clearly visible. Distribution 3(a) has bounded domain with sharp left limit. Distribution $3(\mathrm{~b})$ is also bounded but characterized by the high peak. Distribution 4 has unbounded domain but is otherwise almost similar to distribution 3(a). Distribution 6 is bounded from the left and characterized by a minor 'peak' around $x=2$.

The contours of $\tau_{3}$ and $\tau_{4}$ in different GLD regions are shown in Figures 7, 8 and 9, In the region 3 the contours are rather complicated. From Figure 7 (a) and 7 (c) it can be seen that there are three separate areas where $\tau_{3}$ has negative values. It is also seen that besides the line $\lambda_{3}=\lambda_{4}$, there are two curves where $\tau_{3}=0$. Naturally, the distributions defined by the curves are not symmetric; they just have zero L-skewness.

\section{Conclusion}

We have presented the L-moments of the GLD up to an arbitrary order and studied which values of L-skewness $\tau_{3}$ and L-kurtosis $\tau_{4}$ can be achieved by the GLD. For the symmetric case the boundaries of were derived analytically and in the general case numerical methods were used. It was found that with an exception of the smallest values of $\tau_{4}$, the GLD covers all possible $\left(\tau_{3}, \tau_{4}\right)$ pairs and often there are two or more distributions sharing the same $\tau_{3}$ and 
$\tau_{4}$. The example in Section 4 demonstrates a situation where there are four GLD members sharing the same $\tau_{3}$ and $\tau_{4}$.

We argue that L-moments are natural descriptive statistics for the GLD because they, differently from the central moments, can be expressed in closed form. The existence of the closed form presentation of the GLD L-moments follows from the fact that the inverse distribution function of the GLD is available in closed form and can be integrated. This relation between L-moments and distributions defined by the inverse distribution function is not restricted only to the GLD (Karvanen, 2006). The existence of the first four central moments of the GLD requires that $\lambda_{3}, \lambda_{4}>-1 / 4$ while the existence of L-moment of any order requires only that $\lambda_{3}, \lambda_{4}>-1$. Thus, using the L-moments we can characterize wider subset of the GLD than using the central moments. Even wider subset of the GLD could be characterized using the trimmed L-moments (Elamir and Seheult, 2003).

The results presented in this paper can be utilized in model selection and estimation. The characterization by L-moments gives an insight which $\tau_{3}$ and $\tau_{4}$ are available in each GLD region. For instance, there are symmetric GLD members available from both the region 3 and 4 , if $\tau_{4}>1 / 6$. This kind of information is useful when making the decision whether the GLD is a potential model for certain data. The results are also useful in the estimation of the GLD parameters. The parameters can be estimated directly by method of L-methods or the L-moment estimates may be used as starting values for other estimation methods. In both cases, we can use the L-ratio boundaries to specify the potential GLD regions where we should search for the parameter estimates. The choice between alternative solutions can be based on the type of the domain (bounded/unbounded) or some other additional criterion such as Kolmogorov-Smirnov statistic or L-moment rations $\tau_{5}$ and $\tau_{6}$.

\section{References}

Asquith, W. H., 2006. L-moments and TL-moments of the generalized lambda distributions. Computational Statistics \& Data Analysis, doi: 10.1016/j.csda.2006.07.016.

Bergevin, R. J., 1993. An analysis of the generalized lambda distribution. Master's thesis, Air Force Institute of Technology, available from http://stinet.dtic.mil.

Bigerelle, M., Najjar, D., Fournier, B., Rupin, N., Iost, A., 2005. Application 
of lambda distributions and bootstrap analysis to the prediction of fatigue lifetime and confidence intervals. International Journal of Fatigue 28, 223236.

Corrado, C. J., 2001. Option pricing based on the generalized lambda distribution. Journal of Future Markets 21, 213-236.

Dudewicz, E. J., Karian, Z. A., 2000. Fitting Statistical Distributions: The Generalized Lambda Distribution and Generalized Bootstrap Methods. Chapman \& Hall/CRC Press, Boca Raton, Florida.

Elamir, E. A., Seheult, A. H., 2003. Trimmed L-moments. Computational Statistics \& Data Analysis 43, 299-314.

Fournier, B., Rupin, N., Bigerellle, M., Najjar, D., Iost, A., Wilcox, R., 2006. Estimating the parameters of a generalized lambda distributions. Computational Statistics \& Data Analysis, doi: 10.1016/j.csda.2006.09.043.

Headrick, T. C., Mugdadib, A., 2006. On simulating multivariate nonnormal distributions from the generalized lambda distribution. Computational Statistics \& Data Analysis 50, 3343-3353.

Hosking, J., 1990. L-moments: Analysis and estimation of distributions using linear combinations of order statistics. Journal of Royal Statistical Society B 52 (1), 105-124.

Hosking, J. R. M., 2006. On the characterization of distributions by their Lmoments. Journal of Statistical Planning and Inference 136 (1), 193-198.

Jones, M. C., 2004. On some expressions for variance, covariance, skewness and L-moments. Journal of Statistical Planning and Inference 126, 97-106.

Karian, Z. A., Dudewicz, E. J., 1999. Fitting the generalized lambda distribution to data: A method based on percentiles. Communications in Statistics: Simulation and Computation 28 (3), 793-819.

Karian, Z. A., Dudewicz, E. J., McDonald, P., 1996. The extended generalized lambda distribution system for fitting distributions to data: History, completion of theory, tables, applications, the "final word" on moment fits. Communications in Statistics: Simulation and Computation 25 (3), 611-642. 
Karvanen, J., 2003. Generation of correlated non-Gaussian random variables from independent components. In: Proceedings of Fourth International Symposium on Independent Component Analysis and Blind Signal Separation, ICA2003. pp. 769-774.

Karvanen, J., 2006. Estimation of quantile mixtures via L-moments and trimmed L-moments. Computational Statistics \& Data Analysis 51 (2), 947-959.

Karvanen, J., Eriksson, J., Koivunen, V., 2002. Adaptive score functions for maximum likelihood ICA. Journal of VLSI Signal Processing 32, 83-92.

King, R., 2006. gld: Basic functions for the generalised (Tukey) lambda distribution. $\mathrm{R}$ package version 1.8.1.

King, R., MacGillivray, H., 1999. A startship estimation method for the generalized lambda distributions. Australian and New Zealand Journal of Statistics 41 (3), 353-374.

Lakhany, A., Mausser, H., 2000. Estimating parameters of generalized lambda distribution. Algo Research Quarterly 3 (3), 47-58.

Lampasi, D. A., Di Nicola, F., Podestà, L., 2005. The generalized lambda distribution for the expression of measurement uncertainty. In: Proceedings of IMTC-2005 IEEE Instrumentation and Measurement Technology Conference. pp. 2118-2133.

Öztürk, A., Dale, R., 1985. Least squares estimation of the parameters of the generalized lambda distribution. Technometrics 27 (1), 81-84.

Pal, S., 2005. Evaluation of non-normal process capability indices using generalized lambda distributions. Quality Engineering, 77-85.

R Development Core Team, 2006. R: A Language and Environment for Statistical Computing. R Foundation for Statistical Computing, Vienna, Austria.

URL http://www.R-project.org

Ramberg, J. S., Schmeiser, B. W., 1974. An approximate method for generating asymmetric random variables. Communications of the ACM 17, $78-82$.

Schnute, J., Boers, N., Haigh, R., and others, 2006. PBSmapping: PBS Mapping 2. R package version 2.09. 


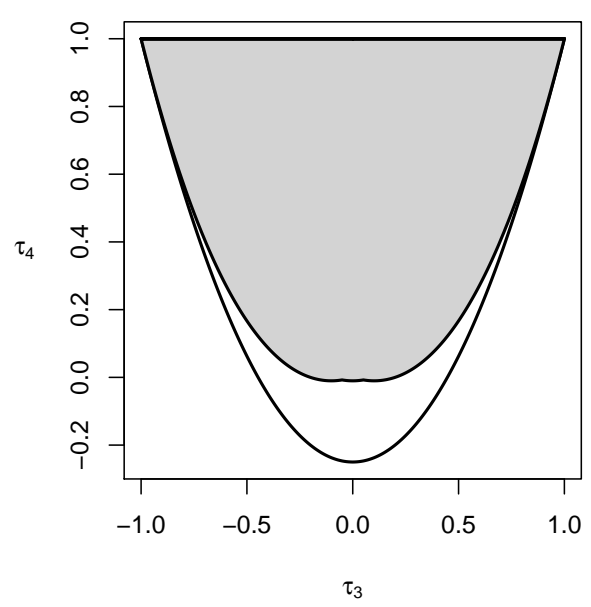

(a) Region 3

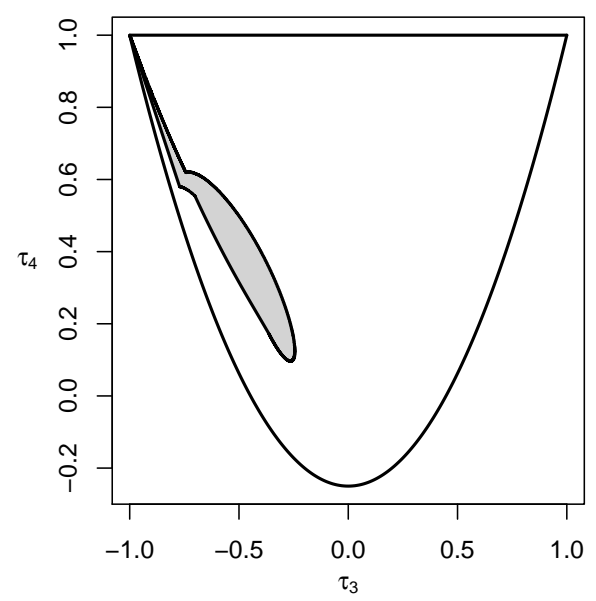

(c) Region 5

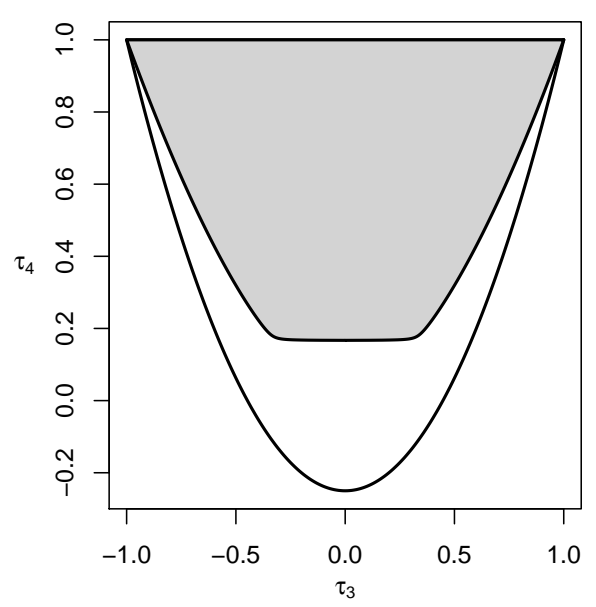

(b) Region 4

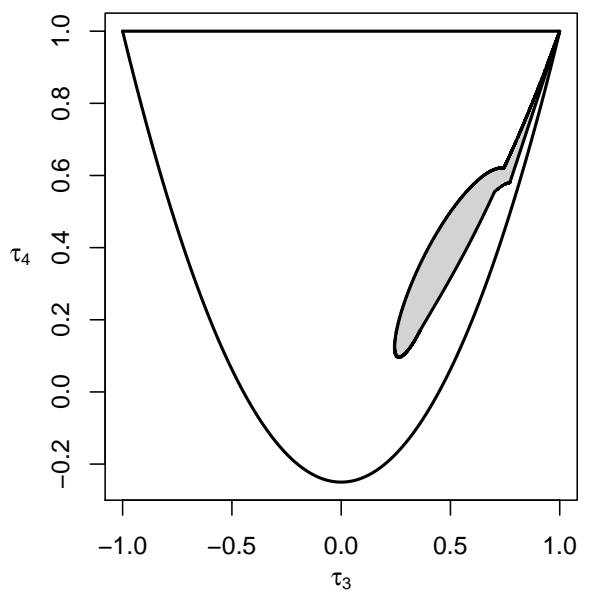

(d) Region 6

Figure 4: Boundaries of the GLD in the $\left(\tau_{3}, \tau_{4}\right)$ space. The shading shows the values of L-skewness and L-kurtosis that are achievable by the GLD. The outer limits present the boundaries for all distributions. 


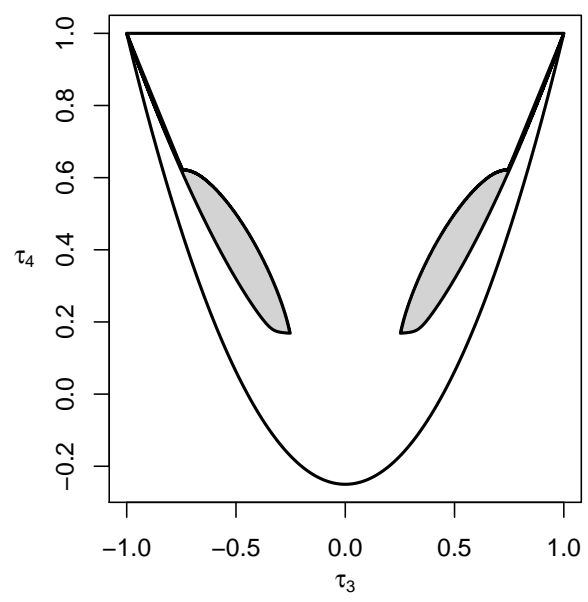

Figure 5: The $\left(\tau_{3}, \tau_{4}\right)$ area where there exist GLD members from the regions 3,4 and 5 (negative L-skewness), or from the regions 3, 4 and 6 (positive L-skewness).

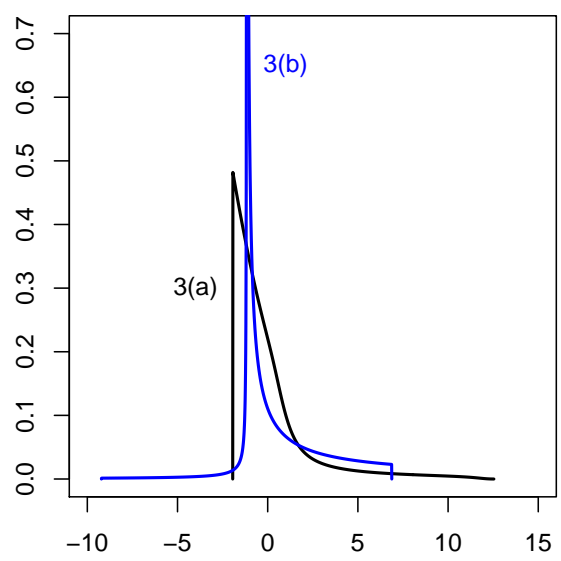

(a) from the region 3

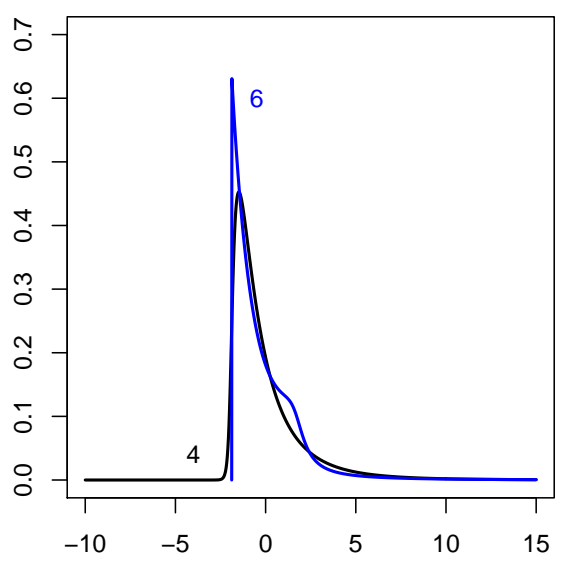

(b) from the regions 4 and 6

Figure 6: Pdfs of four GLDs with $\tau_{3}=0.4$ and $\tau_{4}=0.25$. The parameters of the distributions are presented in Table 1 . 


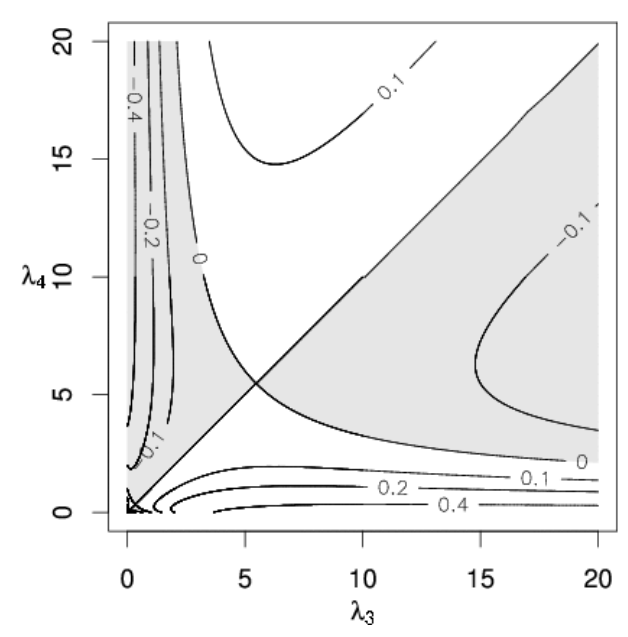

(a) Contours of L-skewness $\tau_{3}$

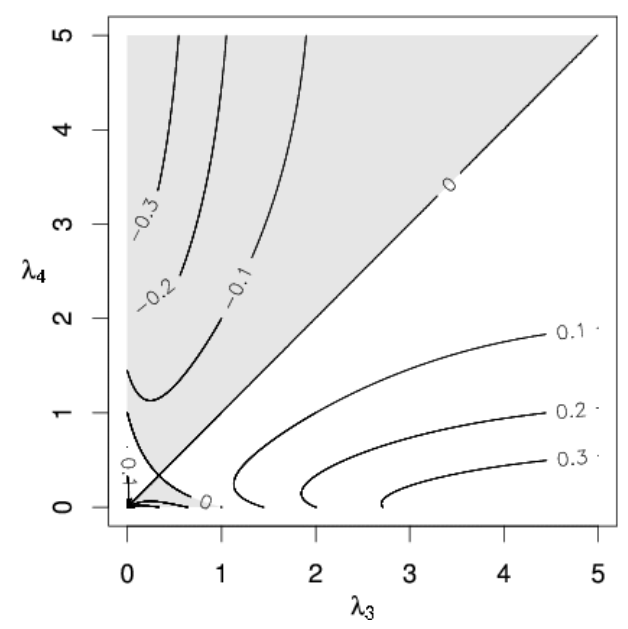

(c) Contours of L-skewness $\tau_{3}$ (zoomed)

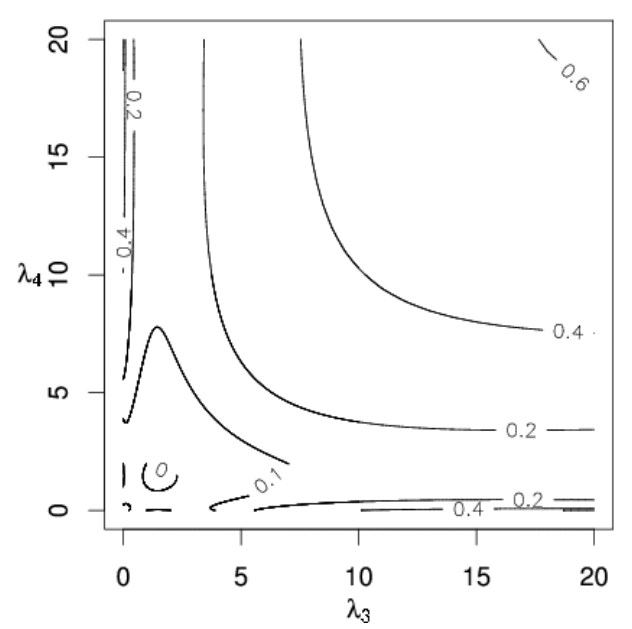

(b) Contours of L-kurtosis $\tau_{4}$

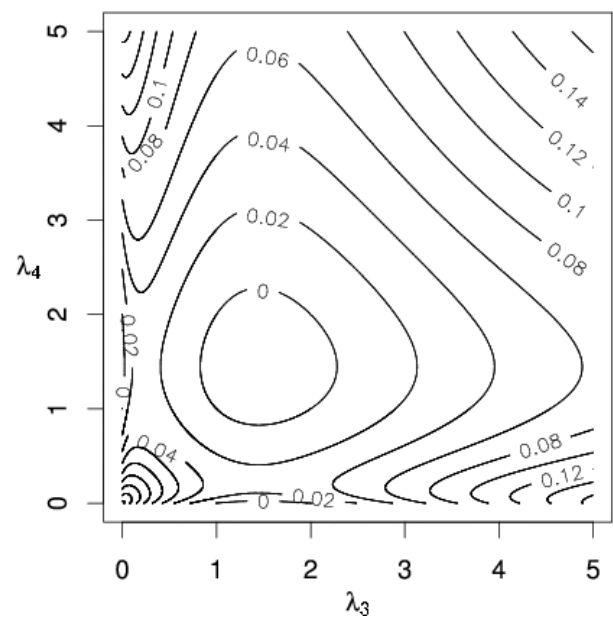

(d) Contours of L-kurtosis $\tau_{4}$ (zoomed)

Figure 7: Contours of $\tau_{3}$ and $\tau_{4}$ in the region 3. The shading indicates negative values of $\tau_{3}$. 


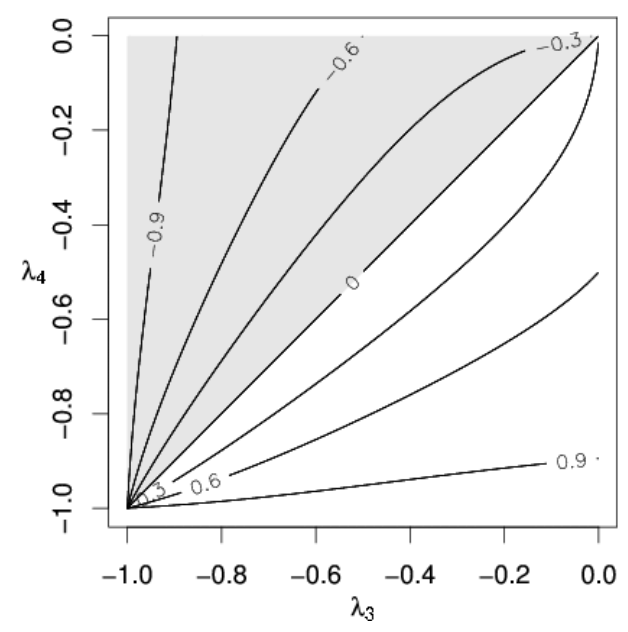

(a) Contours of L-skewness $\tau_{3}$

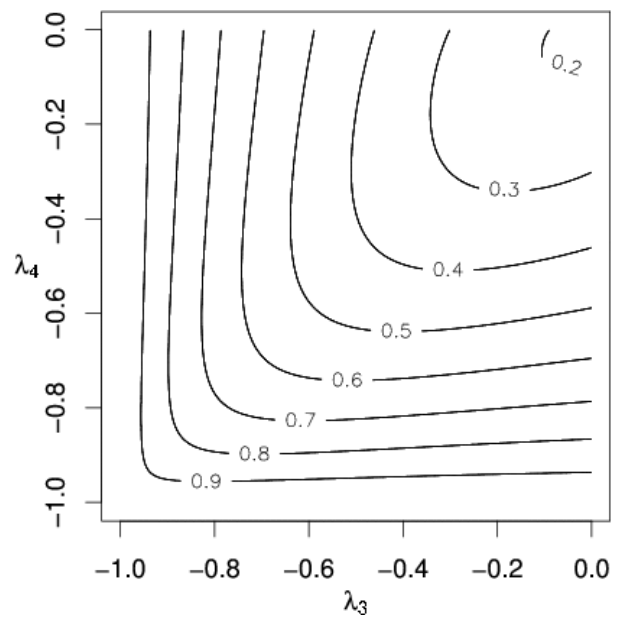

(b) Contours of L-kurtosis $\tau_{4}$

Figure 8: Contours of $\tau_{3}$ and $\tau_{4}$ in the region 4 . The shading indicates negative values of $\tau_{3}$

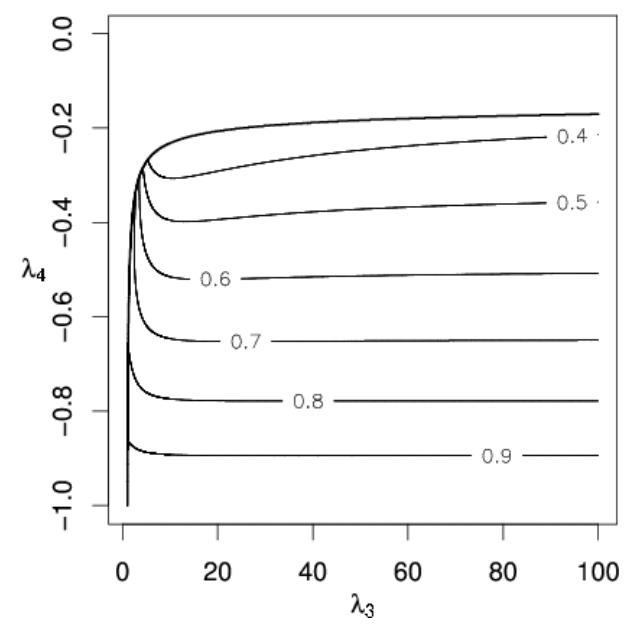

(a) Contours of L-skewness $\tau_{3}$

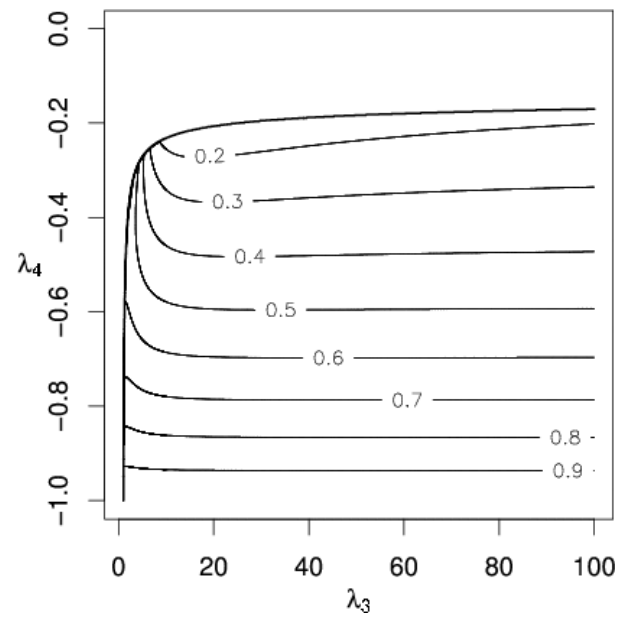

(b) Contours of L-kurtosis $\tau_{4}$

Figure 9: Contours of $\tau_{3}$ and $\tau_{4}$ in the region 6 . 